\title{
Biocoagulación de aguas residuales de industria textilera mediante extractos de Caesalpinia spinosa
}

\section{(Textile wastewater biocoagulation by Caesalpinia spinosa extracts)}

\author{
Andrés Revelo ${ }^{1}$, Diego Proaño ${ }^{2}$, Carlos Banchón ${ }^{3}$
}

\begin{abstract}
Resumen:
La industria textil en Ecuador es todavía motivo de preocupación debido a la inadecuada disposición de sus residuos en los suministros locales de agua. La presente investigación fue llevada a cabo en Pelileo (Tungurahua - Ecuador) donde el agua residual de textileras son descargadas a los cuerpos de agua. Una solución ambientalmente amigable para tratar aguas residuales de textileras con alta carga orgánica es aquí evaluada: un proceso de remediación de biocoagulación fue realizado utilizando extractos de la planta Caesalpinia spinosa conocida como guarango o tara. Se determinó que utilizando extractos de $C$. spinosa para tratar agua residual tiene el mismo efecto estadístico que aplicando un coagulante químico (policloruro de aluminio 15\%). Zeolita activada adsorbió el color residual del agua tratada para obtener una remoción de turbidez más del $90 \%$. Un modelo matemático mostró que la remoción de turbidez entre $50-90 \%$ puede obtenerse aplicando $25-45 \mathrm{~g} / \mathrm{L}$ de extractos de guarango y zeolita por cada $700 \mathrm{~mL}$ de agua residual de textileras. La coagulación natural utilizando extractos de $C$. spinosa produjo $85 \%$ menos lodo que el policloruro de aluminio, y removió altos contenidos de materia orgánica en el agua residual (1050 mg/L) en un $52 \%$.
\end{abstract}

Palabras clave: coagulación; floculación; tara; tanino; turbidez

\begin{abstract}
:
The textile industry in Ecuador is still a matter of concern because of the inappropriate disposal of their effluents into the local water supply. The present research was carried out in Pelileo (Tungurahua-Ecuador) where textile wastewaters are discharged into waterways. An environmentally friendly solution to treat highly contaminated organic textile wastewaters is herein evaluated: a remediation process of biocoagulation was performed using extracts from the Caesalpinia spinosa plant also known as guarango or tara. It was determined that using C. spinosa extracts to treat wastewater has the same statistical effect as when applying a chemical coagulant (polyaluminum chloride 15\%). Activated zeolite adsorbed color residuals from treated water to obtain turbidity removal more than $90 \%$. A mathematical model showed that turbidity removal between $50-90 \%$ can be obtained by applying $25-45 \mathrm{~g} / \mathrm{L}$ of guarango extracts and zeolite per $700 \mathrm{~mL}$ of textile wastewater. The natural coagulation using $C$. spinosa extracts produced $85 \%$ less sludge than polyaluminum chloride, and removed high organic matter content in the wastewater $(1050 \mathrm{mg} / \mathrm{L})$ by $52 \%$.
\end{abstract}

Keywords: coagulation; flocculation; tara; tannin; turbidity

\footnotetext{
${ }^{1}$ Universidad de Las Américas, Ingeniería Ambiental, Quito - Ecuador (arevelo@udlanet.ec)

2 Universidad de Las Américas, Ing. Agroindustrial, Quito - Ecuador (d.proano@udlanet.ec)

${ }^{3}$ Universidad de Las Américas, Ingeniería Ambiental, Quito - Ecuador (carlos.banchon@udla.edu.ec)
} 


\section{Introducción}

En países en desarrollo, un $70 \%$ de aguas residuales se descargan sin tratamiento a cuerpos de agua y aproximadamente dos millones de toneladas de residuos industriales, domésticos y agrícolas son desechados de igual forma en ríos o canales (Corcoran et al., 2010; UNWATER, 2014). En Ecuador, el control ambiental de desechos ha sido muy limitado desde hace décadas. Por ejemplo, en el cantón Pelileo (Tungurahua-Ecuador), a la actualidad, la generación de aguas residuales es el resultado de actividades industriales p.e. lavados de jeans; de esto, se desconocen datos precisos sobre la remediación de efluentes aunque la contaminación en ríos de la zona es latente (El Telégrafo, 2014; I. Municipalidad de Pelileo, 2009). El uso de coagulantes naturales o biocoagulantes extraídos de plantas promueve una alternativa ambientalmente amigable para incentivar la remediación de efluentes industriales como los de Pelileo. Actualmente, extractos de plantas se utilizan para mejorar la calidad del agua en países de África y América Latina (Asrafuzzaman, Fakhruddin, \& Hossain, 2011; Yongabi, 2010). En algunos casos, se ha reemplazado el uso de productos químicos. Por ejemplo, harina de Moringa oleifera se utiliza como un poderoso adsorbente y agente antimicrobiano (Alo et al., 2012; Mangale Sapana, Chonde Sonal, \& Raut, 2012; Pallavi \& Mahesh, 2013; Yarahmadi et al., 2009). Biocoagulantes extraídos de Moringa stenopetala, Caesalpinia spinosa (Sánchez-Martín, BeltránHeredia, \& Gragera-Carvajal, 2011), Cicer arietinum (Chickpea), cactus y goma arábica también han sido investigados. Extractos de guarango (Caesalpinia spinosa) han sido reportados por su potencial adsorbente para el tratamiento de aguas contaminadas con colorantes (Sánchez-Martín, Beltrán-Heredia, \& Gragera-Carvajal, 2011). El guarango o tara es una planta andina que crece en la zona occidental de la cordillera de Los Andes y en valles interandinos de Venezuela, Colombia, Ecuador, Perú, Chile y Bolivia (Chambi et al., 2013; Mancero, 2008). Con los frutos del guarango, se pueden obtener varios productos, de los cuales, los más importantes son extractos de taninos. Los taninos tienen aplicaciones directas en la industria p.e. curtido de pieles, clarificación de vinos y de cerveza, fabricación de plásticos, entre otros (Nieto \& Hidrobo, 2011).

Ante la problemática expuesta, el objetivo del presente trabajo se refiere a la evaluación de un proceso de remediación de aguas residuales de textileras. También se presenta la evaluación de factores operacionales para la dosificación de extractos biocoagulantes obtenidos a partir de Caesalpinia spinosa.

\section{Metodología}

\subsection{Caracterización físico-química del agua}

Las muestras de agua residual $(\mathrm{pH}=8-10)$ fueron tomadas de la empresa textil Dayantex (Barrio El Tambo, cantón Pelileo, provincia de Tungurahua, Ecuador). La turbidez se midió en un turbidímetro (HANNA Instruments) en unidades nefelométricas de turbidez (NTU, Nephelometric 
Turbidity Unit). La demanda química de oxígeno (DQO) en $\mathrm{mg} \mathrm{O}_{2} / \mathrm{L}$ fue determinada mediante espectrofotometría UV-VIS a $620 \mathrm{~nm}$, según el método analítico estándar CP-PEE-A020 (CESAQPUCE 13163-1). El volumen de lodos sedimentables fue determinado mediante el método estándar ASTM 2540-F.

\subsection{Extractos de Caesalpinia spinosa}

Se agregaron $0.1 \mathrm{~g}$ de harina de guarango (Caesalpinia spinosa kuntze) por cada $\mathrm{mL}$ de agua destilada. Se experimentó con un volumen de $500 \mathrm{~mL}$ de solución de guarango. Esta solución se calentó a $135^{\circ} \mathrm{C}$ y se agitó a 210 rpm por 30 min. Para separar los sólidos precipitados, se utilizó un papel filtro Whatman de $125 \mathrm{~mm}$. El líquido filtrado posteriormente se lo diluyó en proporciones volumétricas iguales con metanol concentrado al 90\% (Živković, Mujić, Nikolić, Vidović, \& Mujić, 2009). Esta solución final denominada biocoagulante tuvo una densidad de $1.087 \mathrm{~g} / \mathrm{mL}$.

\subsection{Remediación de aguas residuales de textileras con extractos de $C$. spinosa}

El proceso de remediación consistió de las siguientes operaciones: coagulación-floculación y filtración en lecho de zeolita activada. La coagulación-floculación fue realizada en un equipo Flocumatic (Selecta, España). La dosificación se llevó a cabo según la Tabla 1, donde se indican los intervalos de adición de coagulantes y floculantes, así como las velocidades de mezclado. Se utilizó el coagulante químico policloruro de aluminio al $15 \% \mathrm{~m} / \mathrm{v}$ con densidad $1.261 \mathrm{~g} / \mathrm{mL}$ (PCA, producto comercial VX-50 Dicomsa), biocoagulante de extractos de C. spinosa, y el floculante poliacrilamida $0.1 \% \mathrm{~m} / \mathrm{v}$ (PA, producto comercial, Dicomsa). La remoción final del color que no pudo ser removido con la coagulación, se logró a través de un filtro con zeolita activada. La activación de la zeolita se realizó a $250{ }^{\circ} \mathrm{C}$ durante cuatro horas. Se utilizó una dosis de $21.4 \mathrm{mg}$ de zeolita por cada $\mathrm{mL}$ de agua a decolorar. Las zeolitas fueron del tipo alumino-silicato de elevada hidratación.

Se realizaron los siguientes estudios para determinar las condiciones óptimas del proceso de remediación:

(i) Evaluación del efecto de remoción de turbidez en agua residual de textileras con PCA $15 \%$ versus extractos de C. spinosa, utilizando floculante PA $0.1 \%$, mediante un diseño factorial completamente aleatorio 2 × 2 × 2 según Tabla 2.

(ii) Disminución de la demanda química de oxígeno (DQO) mediante tratamiento con las mejores dosis de biocoagulante y PCA 15\% obtenidas en la primera evaluación (Figura 2).

(iii) Filtración con zeolita activada de agua tratada con las mejores concentraciones de biocoagulante y PCA $15 \%$. También se seleccionan los tratamientos más óptimos de la primera evaluación. 
Tabla 1. Proceso de coagulación-floculación mediante prueba de jarras para un volumen de $0.7 \mathrm{~L}$ de agua residual de la empresa textilera Dayantex.

\begin{tabular}{|c|c|c|}
\hline Etapas & $\begin{array}{c}\text { Intervalos de } \\
\text { tempo (min) }\end{array}$ & Velocidad (rpm) \\
\hline Adición de coagulante & $0-3$ & 200 \\
\hline Adición de floculante & $3-5$ & 200 \\
\hline Floculación & $5-15$ & 40 \\
\hline Sedimentación & $15-20$ & 0 \\
\hline
\end{tabular}

Los factores de estudio y sus niveles (rangos) de experimentación se presentan en la Tabla 2 en función de un volumen de $0.7 \mathrm{~L}$ de agua residual de textilera. Los rangos de experimentación fueron seleccionados en base a pruebas preliminares.

Tabla 2. Factores y niveles para el diseño factorial completamente aleatorio $2 \times 2 \times 2$.

\begin{tabular}{|c|c|c|}
\hline Factores de estudio & Nivel bajo (-1) & Nivel alto (+1) \\
\hline$X_{1}=$ dosis de coagulante PCA 15\% & $9.0 \mathrm{~g} / \mathrm{L}$ & $18.0 \mathrm{~g} / \mathrm{L}$ \\
\hline $\mathrm{X}_{2}=$ dosis de floculante PA $0.1 \%$ & $5.7 \mathrm{~g} / \mathrm{L}$ & $11.4 \mathrm{~g} / \mathrm{L}$ \\
\hline$X_{3}=$ dosis de biocoagulante C. spinosa & $23.3 \mathrm{~g} / \mathrm{L}$ & $45.0 \mathrm{~g} / \mathrm{L}$ \\
\hline
\end{tabular}

En la Tabla 3, los factores y sus respectivos niveles son combinados aleatoriamente para obtener los respectivos tratamientos de coagulación-floculación. La respuesta a evaluar fue la turbidez del agua tratada en unidades nefelométricas y también el volumen de lodo formado $(\mathrm{mL})$. El análisis estadístico fue elaborado con los paquetes computacionales R-project (R Core Team, 2014; Russell, 2014) y JMP (SAS Institute, versión 9.0).

Tabla 3. Matriz de combinaciones entre factores y niveles.

\begin{tabular}{|c|c|c|c|}
\hline Tratamientos & $\mathbf{X}_{\mathbf{1}}$ (PCA 15\%) & $\mathbf{X}_{\mathbf{2}}$ (PA 0.1\%) & $\mathbf{X}_{3}$ (C. spinosa) \\
\hline T1 & $9.0 \mathrm{~g} / \mathrm{L}(-1)$ & $5.7 \mathrm{~g} / \mathrm{L}(-1)$ & 0 \\
\hline T2 & $18.0 \mathrm{~g} / \mathrm{L}(+1)$ & $5.7 \mathrm{~g} / \mathrm{L}(-1)$ & 0 \\
\hline T3 & $9.0 \mathrm{~g} / \mathrm{L}(-1)$ & $11.4 \mathrm{~g} / \mathrm{L}(+1)$ & 0 \\
\hline T4 & $18.0 \mathrm{~g} / \mathrm{L}(+1)$ & $11.4 \mathrm{~g} / \mathrm{L}(+1)$ & $23.3 \mathrm{~g} / \mathrm{L}(-1)$ \\
\hline T5 & 0 & $5.7 \mathrm{~g} / \mathrm{L}(-1)$ & $45.0 \mathrm{~g} / \mathrm{L}(+1)$ \\
\hline T6 & 0 & $5.7 \mathrm{~g} / \mathrm{L}(-1)$ & $23.3 \mathrm{~g} / \mathrm{L}(-1)$ \\
\hline T7 & 0 & $11.4 \mathrm{~g} / \mathrm{L}(+1)$ & $45.0 \mathrm{~g} / \mathrm{L}(+1)$ \\
\hline T8 & 0 & $11.4 \mathrm{~g} / \mathrm{L}(+1)$ & \\
\hline
\end{tabular}




\section{Resultados}

En la Figura 1, se presentan los resultados de turbidez del agua tratada con PCA 15\%, biocoagulante de guarango y PA $0.1 \%$ de acuerdo con las combinaciones de factores y niveles de la Tabla 3. Se presenta también, la turbidez inicial del agua residual (Blanco). Con estos resultados, en la siguiente sección, se presenta un análisis estadístico para evaluar la influencia de las dosis altas y bajas de cada variable en el porcentaje de remoción de turbidez y DQO.

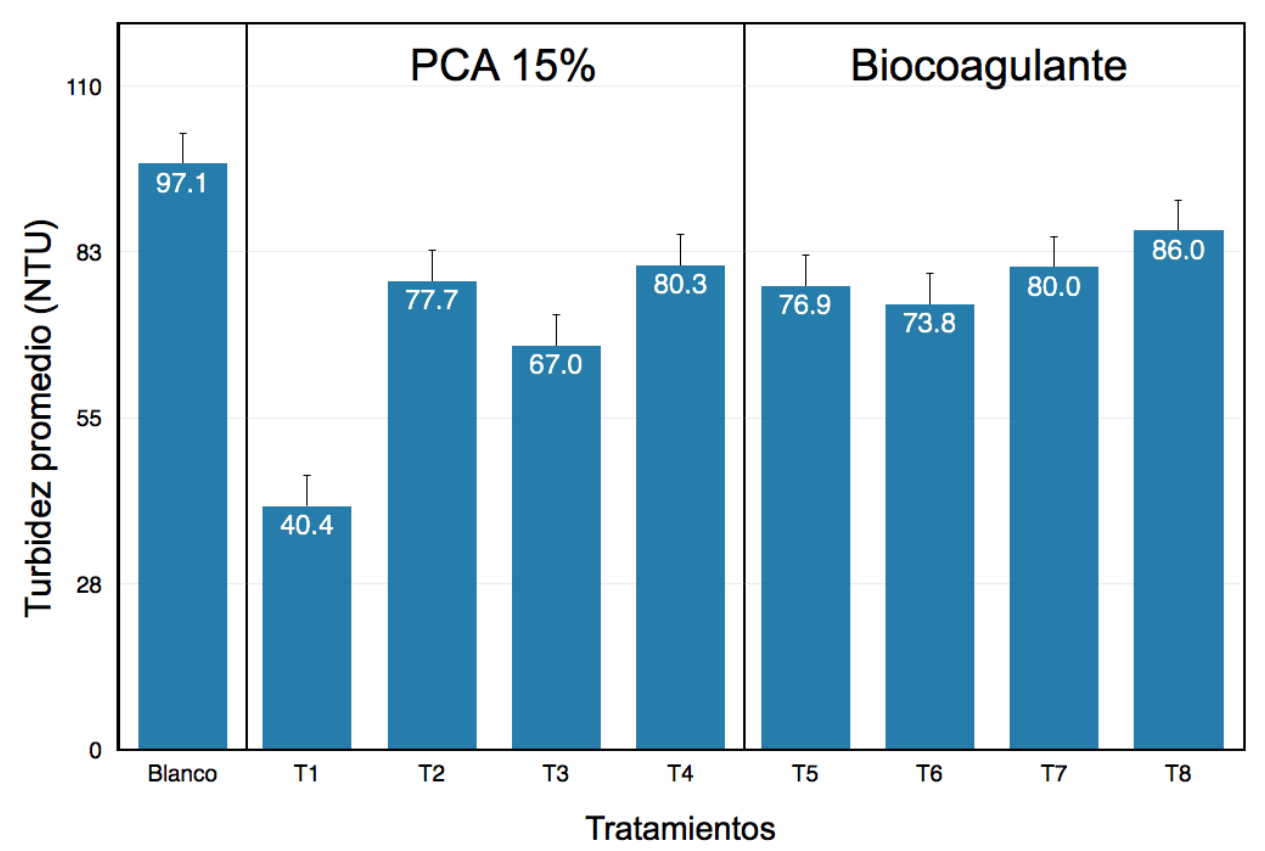

Figura 1. Turbidez promedio antes y después del tratamiento con policloruro de aluminio $15 \%$ (PCA), biocoagulante (C.spinosa) y floculante poliacrilamida $0.1 \%$. Donde, T1-T4 son tratamientos con PCA $15 \%$ y T5-T8 son tratamientos con biocoagulante; Blanco = agua residual de industria textilera; Réplicas, $n=3$.

En función de los resultados de la primera evaluación (Figura 1), se eligieron los tratamientos 1 y 6 para determinar el porcentaje de remoción de DQO (Figura 2). El tratamiento 1, en el que se utilizó una concentración de $9.0 \mathrm{~g} / \mathrm{L}$ de PCA $15 \%$ y $5.7 \mathrm{~g} / \mathrm{L}$ de PA $0.1 \%$, se obtuvo una remoción de turbidez de 58\%; es decir, los niveles bajos de concentración de químicos. El tratamiento 6 , en el que se utilizó una concentración de $45.0 \mathrm{~g} / \mathrm{L}$ de biocoagulante y $5.7 \mathrm{~g} / \mathrm{L}$ de PA $0.1 \%$, se obtuvo una remoción de $24 \%$ de turbidez; es decir, más concentración de biocoagulante y menos de floculante.

La demanda química de oxígeno a través del tratamiento con biocoagulante y PCA $15 \%$ se presenta en la Figura 2, en función del valor máximo permisible por la normativa ambiental ecuatoriana según el Texto Unificado Legislación Secundaria, Medio Ambiente de Ecuador (TULSMA). 


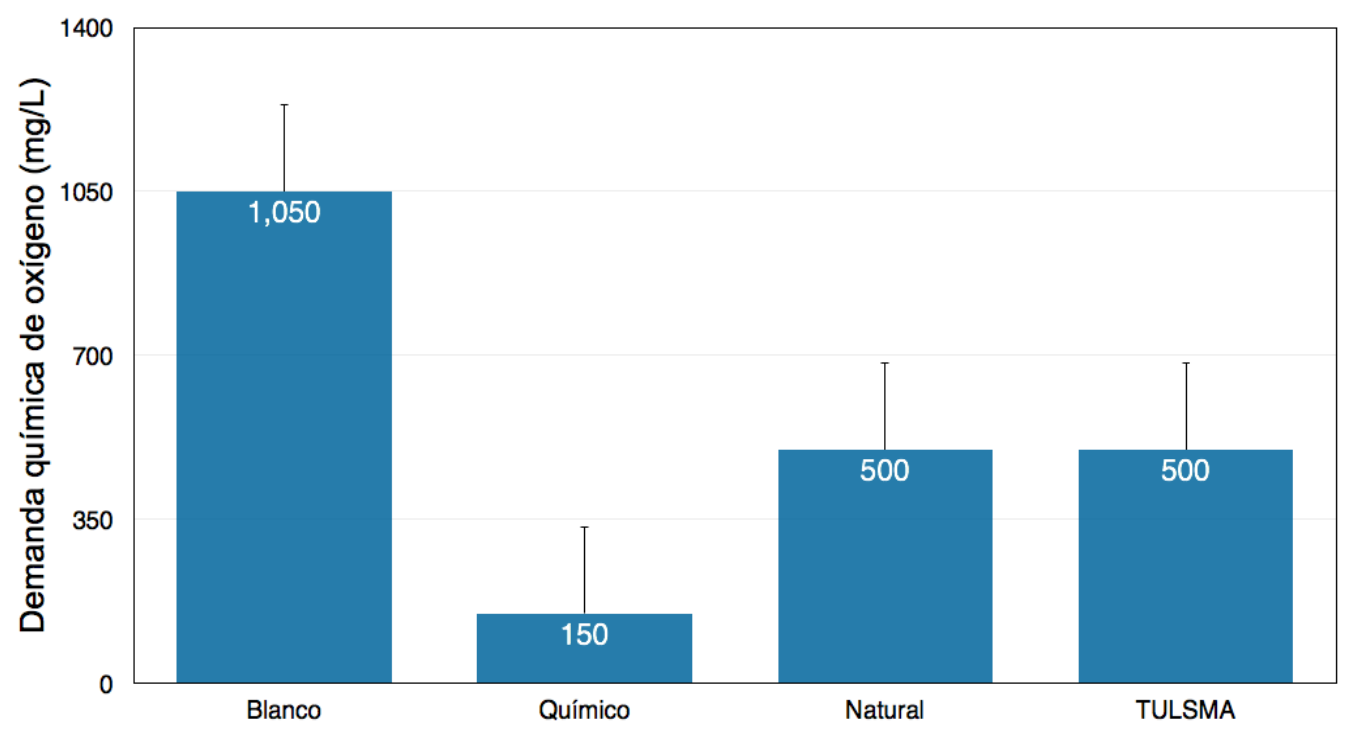

Figura 2. Demanda química de oxígeno en $\mathrm{mg} \mathrm{O}_{2} / \mathrm{L}$ de tratamientos (Químico) con concentración $9.0 \mathrm{~g} / \mathrm{L}$ de PCA $15 \%$ y $5.7 \mathrm{~g} / \mathrm{L}$ de PA $0.1 \%$ en el agua residual y (Natural) con concentración de $45.0 \mathrm{~g} / \mathrm{L}$ de biocoagulante y $5.7 \mathrm{~g} / \mathrm{L}$ de PA $0.1 \%$ en el agua residual. Donde (Blanco) es la muestra de agua residual de textilera sin tratar, y (TULSMA) es el límite máximo permisible de DQO en efluentes al sistema de alcantarillado público.

Luego de la coagulación-floculación, se procedió a filtrar el agua con zeolita activada para remover trazas de color (Figura 3). Para el agua proveniente del tratamiento 1, el tratamiento químico, se obtuvo una turbidez final de 11.8 NTU. Para el agua proveniente del tratamiento 6, el tratamiento químico, se obtuvo una turbidez final de 6.4 NTU.

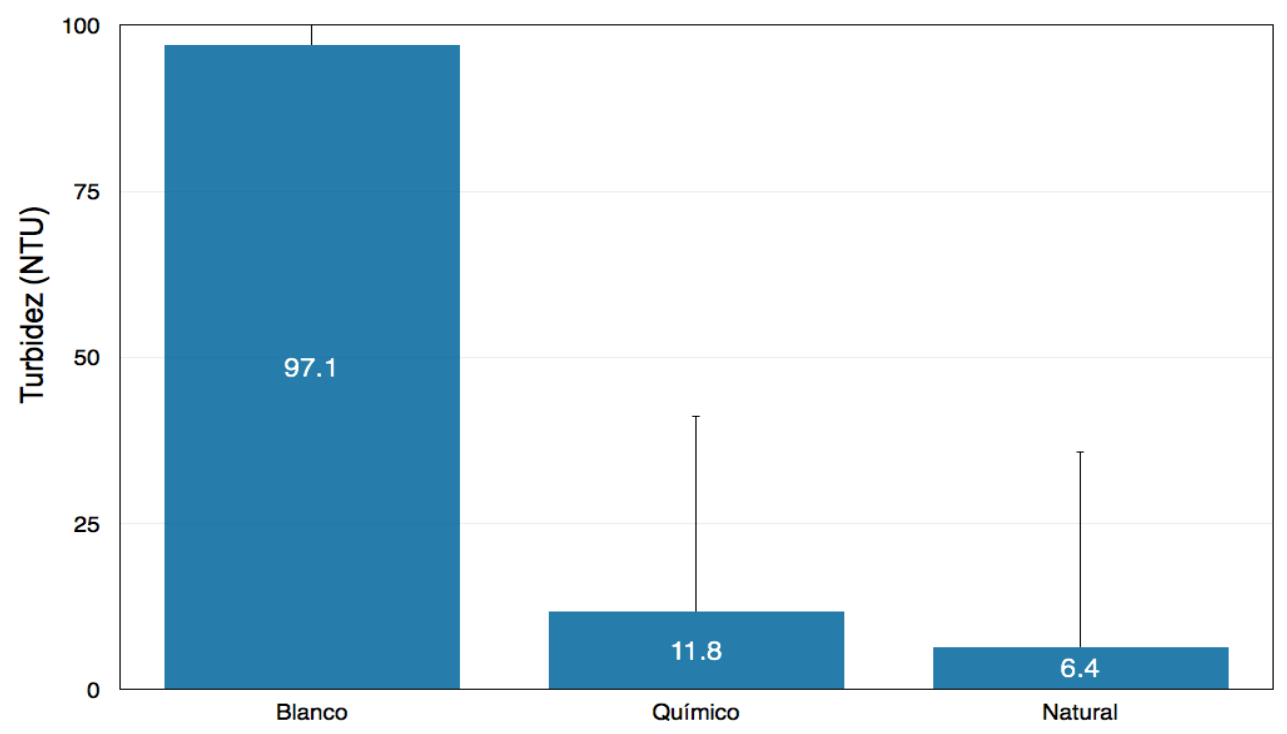

Figura 3. Turbidez del agua tratada con zeolita. Donde (Blanco) muestra de agua residual de textilera sin tratar, (Químico) agua tratada previamente con $9.0 \mathrm{~g} / \mathrm{L}$ de PCA $15 \%$ y $5.7 \mathrm{~g} / \mathrm{L}$ de PA $0.1 \%$ y (Natural) agua tratada previamente con $45.0 \mathrm{~g} / \mathrm{L}$ de biocoagulante y $5.7 \mathrm{~g} / \mathrm{L}$ de PA $0.1 \%$. 


\section{Discusión}

Según la Figura 1, la remoción de turbidez debido al PCA 15\% fue entre 17-58\%, mientras que con biocoagulante entre $11-24 \%$. La filtración con zeolita activada removió hasta 93.4\% de turbidez (Figura 3). Para el agua proveniente del tratamiento 1, el tratamiento químico, el filtro de zeolita removió turbidez hasta un $87.9 \%$. Mientras que para el agua proveniente del tratamiento 6 , el tratamiento natural, el filtro de zeolita removió turbidez hasta un 93.4\%.

Según la Figura 2, la remoción de demanda química de oxígeno mediante el coagulante PCA 15\% llega a un $85 \%$, mientras que el biocoagulante alcanza una remoción del 52\%. El Texto Unificado Legislación Secundaria, Medio Ambiente de Ecuador (TULSMA) establece que el límite máximo permisible de DQO en efluentes al sistema de alcantarillado público es de $500 \mathrm{mg} / \mathrm{L}$; es decir, el agua tratada con extractos de guarango en efecto cumple con esta normativa. Por lo tanto, se constata el potencial coagulante de extractos de $C$. spinosa para la remoción de una alta carga de materia orgánica de $1050 \mathrm{mg} \mathrm{O} / \mathrm{L}$.

En la Figura 4 se muestra el efecto estadístico de la adición de los coagulantes PCA 15\% y extracto de guarango en función de la remoción de turbidez. Según el análisis de varianza (Figura 4), la remoción de turbidez con los dos coagulantes es estadísticamente significativa $(p<0.05)$. Esto sugiere que, en efecto los extractos de guarango pueden utilizarse para la remoción de contaminación coloidal. La remoción de contaminantes del agua residual de textileras fue ya reportada por Sánchez-Martín, Beltrán-Heredia, \& Gragera-Carvajal (2011); no obstante, en el presente estudio se verifica que extractos de $C$. spinosa también remueven un alto grado de turbidez (97.1 NTU) y materia orgánica (1050 $\left.\mathrm{mg} \mathrm{O}_{2} / \mathrm{L}\right)$.
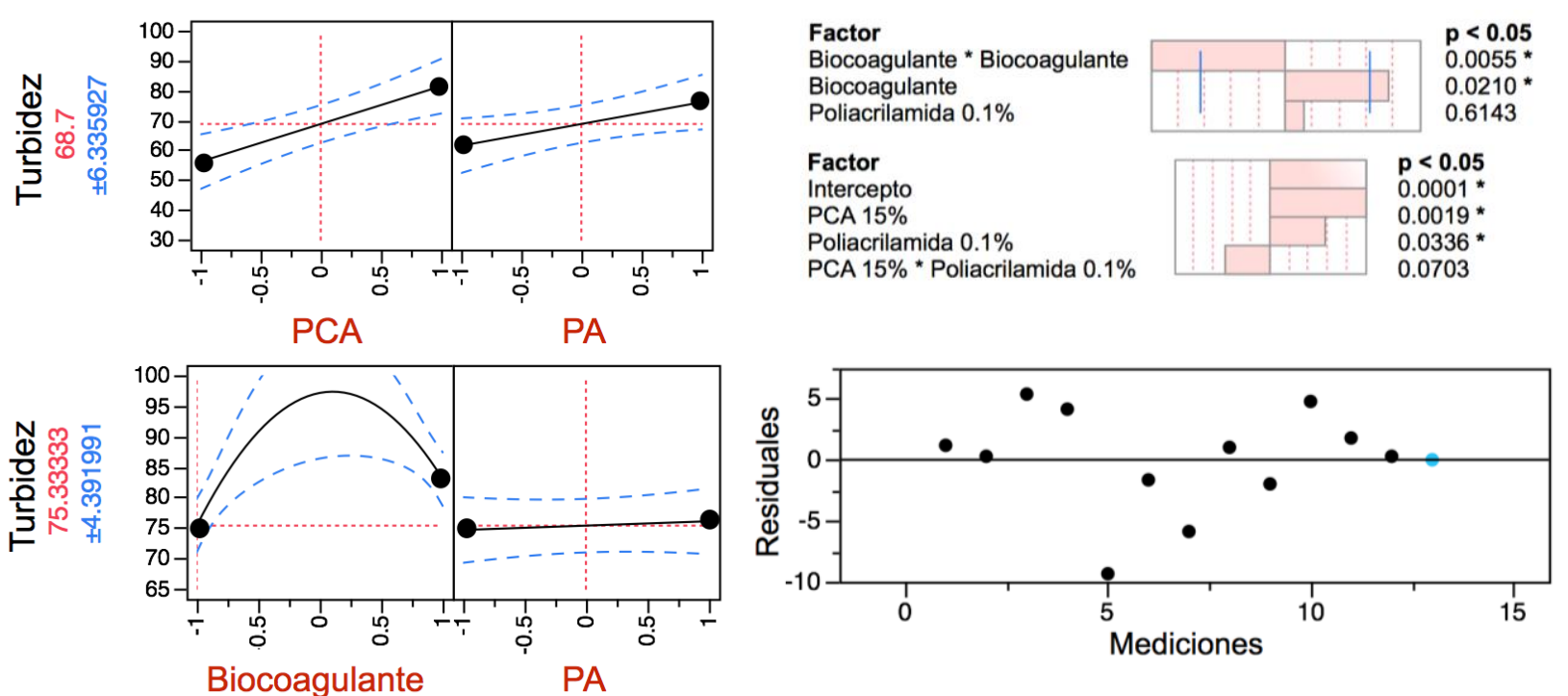

Figura 4. Efectos principales de los factores de estudio (PCA 15\%, biocoagulante y PA $0.1 \%$ ) en función de su residualidad y análisis de la varianza con un $\mathrm{p}<0.05$. 
La goma o endospermo del guarango posee las características propias de las gomas vegetales: actúa como espesante, aglomerante y estabilizador (Nieto e Hidrobo, 2011). Es muy importante recalcar esta propiedad, ya que se la sugiere como parte del mecanismo de coagulación: el de absorción. Además, también se sugiere un mecanismo de protonación producida por la presencia de polifenoles en extractos del guarango, debido a la alta concentración de taninos en el guarango de $40-60 \% \mathrm{~m} / \mathrm{v}$ (Bellotti, del Amo, \& Romagnoli, 2012). El objetivo de añadir cargas positivas es desestabilizar los coloides aniónicos de carga negativa presentes en el agua residual (Beltrán, Sánchez, Gómez y Dávila, 2010). La presencia de sales de hierro en la composición de taninos, también es otra razón para la sedimentación de coloides contaminantes de las aguas residuales; esto es, debido a que el hierro (III) del tanino forma iones complejos que generan hidrólisis de moléculas de agua aportando así cargas positivas al medio. Así, los coloides cargados negativamente son desestabilizados formándose lodos residuales. Esto se comprueba mediante el siguiente razonamiento en base a los resultados experimentales de la presente investigación: si el efecto de remoción de contaminación coloidal y disuelta mediante monómeros de aluminio es positivo (Figuras 1 y 2), entonces el agua residual se compone de contaminación con cargas eléctricas negativas. Si extractos de guarango también tienen efectos positivos de remoción de contaminación coloidal y disuelta, entonces los extractos de guarango disponen de un carácter electropositivo en la desestabilización coloidal, siguiendo mecanismos de neutralización de cargas.

Un modelo matemático realizado en R-project ( $R$ Core Team, 2014; Russell, 2014) mediante el paquete RSM, incorporando el efecto de filtración con zeolita activada, se presenta en la Figura 5 los porcentajes a los cuales llegaría la remoción de turbidez mediante PCA 15\%, PA 0.1\% y extractos de guarango. Estos porcentajes oscilan entre 50-90\%. Estudios sobre coagulación con productos naturales reportan eficiencias de remoción de turbidez de entre 49.71 hasta $95.89 \%$ con extractos de Acacia mearnsii, Moringa oleifera, Dolichos lablab y Cicer arietinum (Asrafuzzaman, Fakhruddin, \& Hossain, 2011; Beltrán-Heredia, Sánchez-Martín, \& RodríguezSánchez, 2011; Kazi \& Virupakshi, 2013).

En referencia a la remoción de lodos, el uso de biocoagulante produjo $85 \%$ menos volumen de lodos que mediante el uso de PCA 15\% (Figura 6). Experimentalmente se demostró que el grado de compactación de los lodos generados en la biocoagulación es mayor que cuando se utiliza policloruro de aluminio, en concordancia con lo manifestado por Alo, Anyim, \& Elom (2012) sobre el volumen de lodos generados por la alumina. Cabe recalcar la relevancia de estudios enfocados a la calidad de compactación de lodos en función de su fácil remoción en sedimentadores de plantas de tratamiento. 

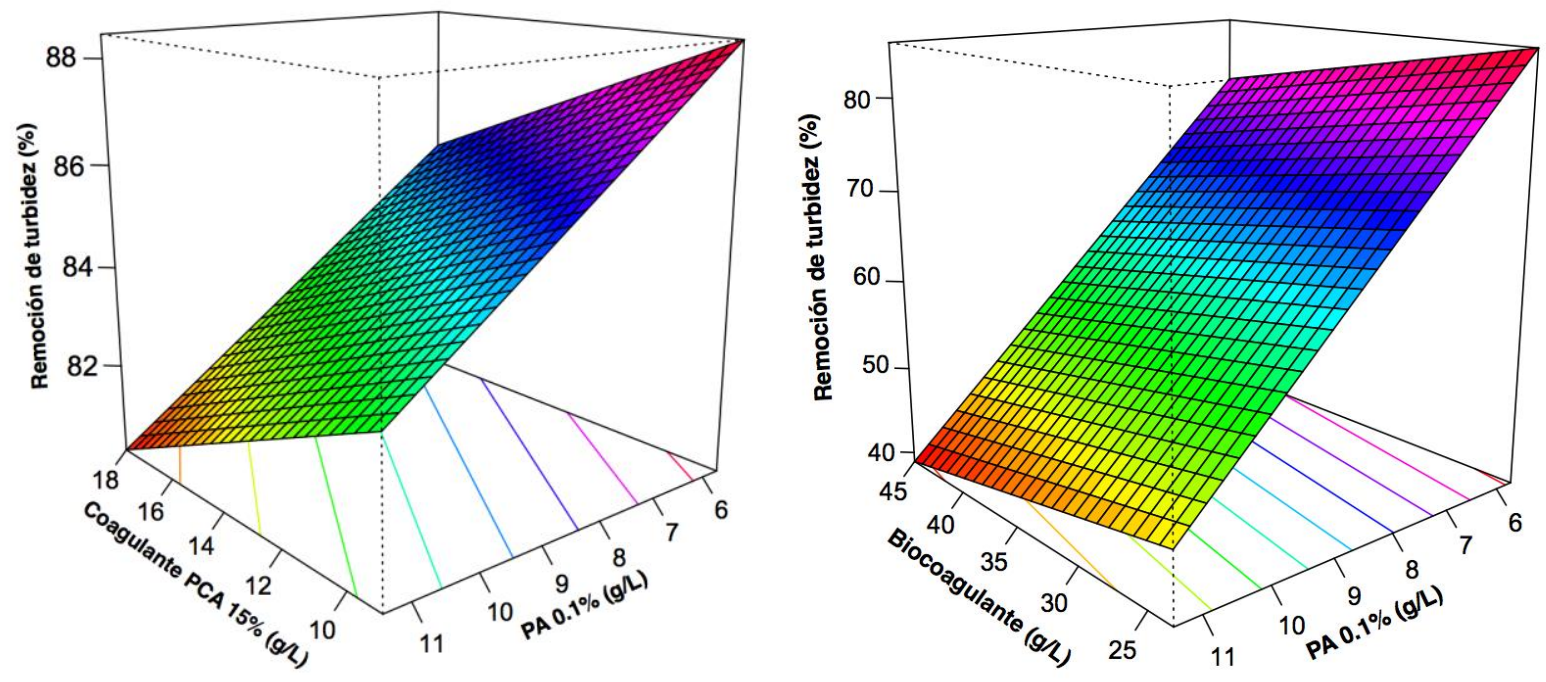

Figura 5. Modelo de superficie de respuesta de remoción de turbidez en función de la concentración de biocoagulante, coagulante PCA 15\%, floculante PA $0.1 \%$ y zeolita activada.
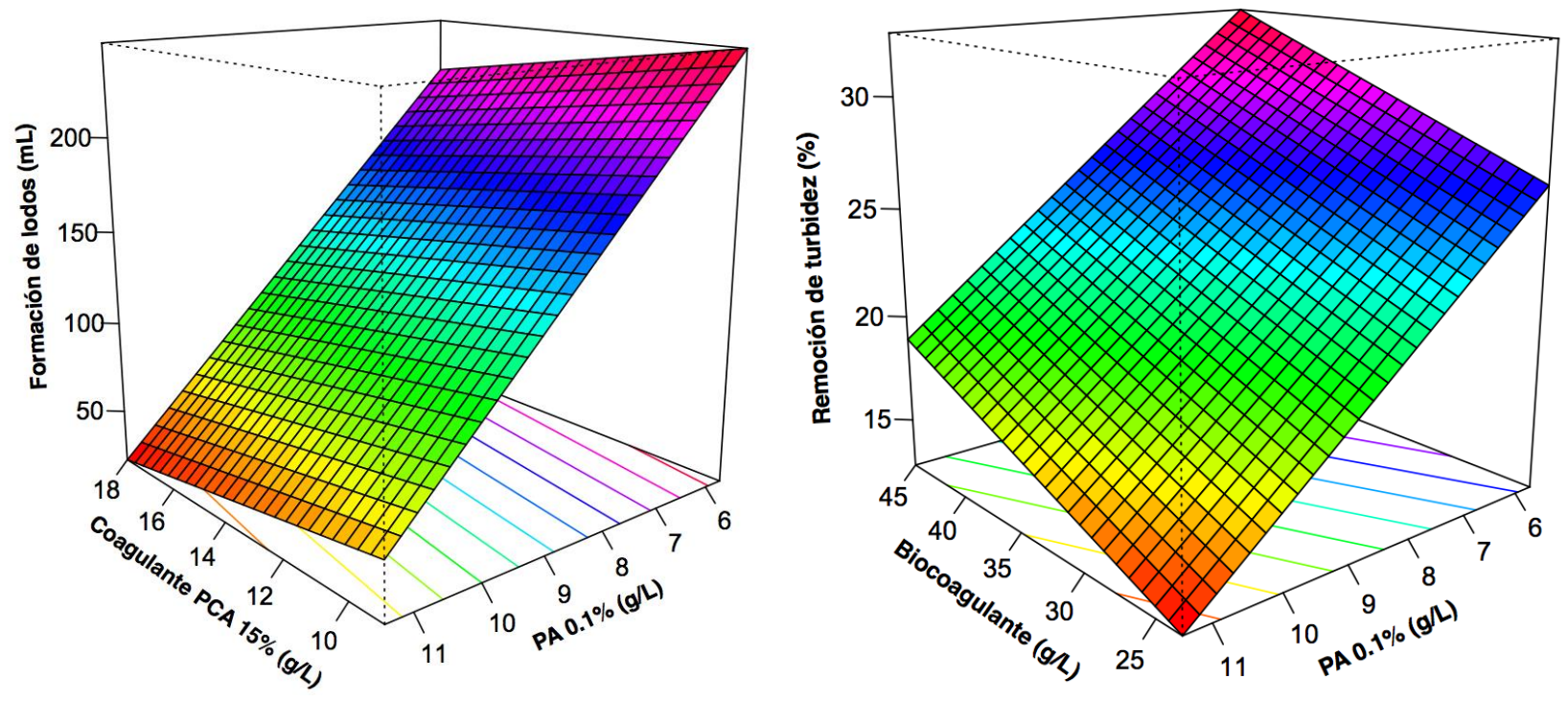

Figura 6. Modelo de superficie de respuesta para la formación de lodos debido al tratamiento con biocoagulante, PCA $15 \%$ y floculante PA $0.1 \%$.

\section{Conclusiones y Recomendaciones}

El proceso de tratamiento de aguas residuales provenientes de una industria textilera consistió en

(i) coagulación-floculación del agua residual, (ii) sedimentación de lodos, (iii) remoción de remanentes colorantes mediante zeolita activada; para este proceso no fue necesario la regulación ni del $\mathrm{pH}$, ni conductividad eléctrica del agua residual. 
El presente estudio revela el potencial de aplicación de extractos biocoagulantes de C. spinosa para la remoción de altas concentraciones de materia orgánica (1050 mg $\mathrm{O}_{2} / \mathrm{L}$ ). No obstante, grados de remoción de turbidez hasta $24 \%$ y DQO hasta $52 \%$, con $45.0 \mathrm{~g} / \mathrm{L}$ de biocoagulante y $5.7 \mathrm{~g} / \mathrm{L}$ de PA $0.1 \%$ sugieren una optimización del proceso de extracción de taninos del guarango.

Se recomienda la optimización en la extracción y caracterización de taninos de plantas endémicas de Ecuador con características similares a las de $C$. spinosa, dado a su potencial de neutralización de cargas negativas y carácter adsorbente de materia orgánica en aguas residuales. Esto como una opción de bajo coste y amigable con el ambiente. La aplicación de biocoagulantes es de gran relevancia en países donde las plantas de tratamiento tecnificadas y automatizadas todavía no forman parte del manejo integral de contaminantes.

\section{Bibliografía}

Alo, M. N., Anyim, C., \& Elom, M. (2012). Coagulation and Antimicrobial Activities of Moringa oleifera Seed Storage at $3^{\circ} \mathrm{C}$ Temperature in Turbid Water. Advances in Applied Science Research, 3(2), 887-894.

Asrafuzzaman, M., Fakhruddin, A. N. M., \& Hossain, M. A. (2011). Reduction of Turbidity of Water Using Locally Available Natural Coagulants. ISRN Microbiology, 2011, 1-6. doi:10.5402/2011/632189

Bellotti, N., del Amo, B., \& Romagnoli, R. (2012). Caesalpinia Spinosa Tannin Derivatives for Antifouling Formulations. Procedia Materials Science, 1, 259-265. doi:10.1016/j.mspro.2012.06.035

Beltrán-Heredia, J., Sánchez-Martín, J., \& Rodríguez-Sánchez, M. T. (2011). Textile wastewater purification through natural coagulants. Applied Water Science, 1(1-2), 2533. doi:10.1007/s13201-011-0005-2

Chambi, F., Chirinos, R., Pedreschi, R., Betalleluz-Pallardel, I., Debaste, F., \& Campos, D. (2013). Antioxidant potential of hydrolyzed polyphenolic extracts from tara (Caesalpinia spinosa) pods. Industrial Crops and Products, 47, 168-175. doi:10.1016/j.indcrop.2013.03.009

Corcoran, E., Nellemann, C., Baker, E., Bos, R., Osborn, D., \& Savelli, H. (2010). Sick water?: the central role of wastewater management in sustainable development : a rapid response assessment. Arendal, Norway: UNEP/GRID-Arendal.

El Telégrafo. (2014). El Parque Industrial de Pelileo proyecta 58 hectáreas. Tomado de http://www.telegrafo.com.ec/regionales/regional-centro/item/el-parque-industrial-depelileo-proyecta-58-hectareas.html 
I. Municipalidad de Pelileo. (2009). Plan estratégico de desarrollo de la parroquia García Moreno (Reporte).

Kazi, T., \& Virupakshi, A. (2013). Treatment of Tannery Wastewater Using Natural Coagulants. Development, 2(8). Retrieved from http://ijirset.com/upload/august/29A_Treatment.pdf

Mancero, L. (2008). La Tara (Caesapinia spinosa) en Perú, Bolivia y Ecuador: Análisis de la cadena productiva en la región. Editorial ECOBONA. Tomado de http://www.asocam.org/portal/node/42571

Mangale Sapana, M., Chonde Sonal, G., \& Raut, P. D. (2012). Use of Moringa oleifera (Drumstick) seed as natural absorbent and an antimicrobial agent for ground water treatment. Research Journal of Recent Sciences, 2277, 2502.

Nieto, C., \& Hidrovo, G. (2011). La cadena agro - productiva de guarango, (Caespinia spinosa kuntze), elementos que resaltan su competitividad. Fundación Desde el Surco, SENESCYT. Tomado de http://repositorio.educacionsuperior.gob.ec/handle/28000/137

Pallavi, N., \& Mahesh, S. (2013). Feasibility Study of Moringa oleifera as a Natural Coagulant for the Treatment of Dairy Wastewater. International Journal of Engineering Research, 2(3), 200-202.

R Core Team. (2014). R: A language and environment for statistical computing. $\mathrm{R}$ Foundation for Statistical Computing, Vienna, Austria. Retrieved from http://www.Rproject.org/

Russell, V. L. (2014). Response-Surface Methods in R, Using rsm. Retrieved from http://www.jstatsoft.org/v32/i07/

Sánchez-Martín, J., Beltrán-Heredia, J., Delgado-Regaña, A., Rodríguez-González, M. A., \& Rubio-Alonso, F. (2013). Optimization of tannin rigid foam as adsorbents for wastewater treatment. Industrial Crops and Products, 49, 507-514. doi:10.1016/j.indcrop.2013.05.029

Sánchez-Martín, J., Beltrán-Heredia, J., \& Gibello-Pérez, P. (2011). Adsorbent biopolymers from tannin extracts for water treatment. Chemical Engineering Journal, 168(3), 12411247. doi:10.1016/j.cej.2011.02.022

Sánchez-Martín, J., Beltrán-Heredia, J., \& Gragera-Carvajal, J. (2011). Caesalpinia spinosa and Castanea sativa tannins: A new source of biopolymers with adsorbent capacity. Preliminary assessment on cationic dye removal. Industrial Crops and Products, 34(1), 1238-1240. doi:10.1016/j.indcrop.2011.03.024 
UNWATER. (2014). Water pollution. The United Nations Inter-Agency Mechanism on All Freshwater Related Issues, including Sanitation. Retrieved from http://www.unwater.org/statistics/statistics-detail/en/c/211800/

Yarahmadi, M., Hossieni, M., Bina, B., Mahmoudian, M. H., Naimabadie, A., \& Shahsavani, A. (2009). Application of Moringa oleifera seed extract and poly aluminium chloride in water treatment. World Appl Sci J, 7(8), 962-967.

Yongabi, K. A. (2010). Biocoagulants for Water and Waste Water Purification: a Review. International Review of Chemical Engineering-Rapid Communications, 2(3), 444-458.

Živković, J., Mujić, I., Nikolić, G., Vidović, S., \& Mujić, A. (2009). Extraction and analysis of condensed tannins in Castanea sativa mill. Journal of Central European Agriculture, 10(3), 283-0. 